\title{
Uso problemático de las redes sociales en estudiantes universitarios
}

\author{
Problematic use of social networks in university \\ students \\ José SÁNCHEZ-RODRÍGUEZ, Julio RUIZ-PALMERO \\ y Enrique SÁNCHEZ-RIVAS \\ Universidad de Málaga
}

Recibido: Septiembre 2014

Aceptado: Octubre 2014

\begin{abstract}
Resumen
Los avances que están produciéndose en el ámbito académico con el surgimiento de herramientas de la Web 2.0 y el empleo masivo por parte de los estudiantes de las redes sociales para comunicarse entre ellos, está haciendo que el panorama educativo se encuentre ante unos desafíos a los que tiene que dar respuesta. La investigación que aquí se presenta tuvo como objetivo principal analizar el estado del empleo de la redes sociales por parte alumnado universitario, así como los posibles malos hábitos y usos problemáticos de las mismas. Se utilizó como instrumento de recogida de información un cuestionario “ad hoc” con un total de 23 ítems. Podemos concluir que el alumnado en general no posee malos hábitos en el empleo de las redes sociales, igualmente los resultados obtenidos ponen de manifiesto que su utilización no está plenamente integrada en las instituciones universitarias de educación superior, así como que los estudiantes no las utilizan como herramienta fundamental para las resolución de cuestiones académicas.
\end{abstract}

Palabas clave: redes sociales (en línea), educación superior, internet, hábitos, web 2.0.

\begin{abstract}
The developments taking place in the academic field with the emergence of Web 2.0 tools and the massive use by students of social networks to communicate with each other is making the educational landscape face some challenges which must be answered. The research presented was mainly intended to analyze the status of the use of social media by college students as well as possible bad habits and problematic uses therefore. An "ad hoc" questionnaire with a total of 23 items was used as a gathering information tool. We conclude that students generally do not have bad habits in the use of social networks, also the results show that their use is not fully integrated into the institutions and students do not use them as a fundamental tool for academic resolution issues.
\end{abstract}

Keywords: social network (online), higher education, internet, habits, web 2.0. 
A lo largo de la historia han ido apareciendo tecnologías que han introducido formas de comunicación más solitarias y menos controladas. Hoy en día, la generalización del uso de Internet y el acceso a dispositivos de menor tamaño y mayor accesibilidad ha hecho surgir una gran preocupación por su uso y por algunos efectos que están produciendo en las conductas de los usuarios.

Las etiquetas "adicción a Internet”, "trastorno de adicción a Internet”, “uso patológico de Internet”, “trastorno de dependencia de la red”, “ciberadicción”, etc. se han utilizado para describir el mismo concepto: un uso inadecuado de Internet que produce descuido de otras áreas de la vida (Widyanto y Griffiths, 2006), dificultades para concentrarse y síndrome de abstinencia.

El uso de Internet es muy elevado en la juventud, situándose en la población de 11 a 20 años en el 98\% (Fundación Pfizer, 2009), disponiendo para ello en la Comunidad de Madrid los jóvenes de 14 a 18 años, entre otros dispositivos, de ordenador con acceso a Internet en el domicilio (99\%) o en su habitación para uso individual (70\%) (Rodríguez, Mejías, y Menéndez, 2012). Por tanto es un hecho incuestionable el alto grado de uso que hacen jóvenes y adolescentes de las tecnologías de la información y la comunicación (TIC).

El porcentaje de internautas que manifiestan tener problemas con Internet no supera el 10\% (Carbonell, Fúster, Chamarro, y Oberst, 2012; Munoz-Rivas, Fernandez, y Gamez-Guadix, 2010), no obstante reconocen efectos preocupantes como un mayor deseo de estar conectado, pérdida de noción del tiempo de conexión, sentimientos de culpa, mentir a familiares sobre el tiempo de conexión, cambios en los hábitos de salud, interferencia a nivel social y/o familiar, pérdida de tiempo laboral, académico o de descanso (Gracia, Vigo, Fernández, y Marcó, 2002; Munoz-Rivas et al., 2010; Young, 1998), una disminución de la autoestima (Wang et al., 2013), etc. Por su parte, individuos solitarios y deprimidos pueden desarrollar una preferencia por la interacción social en línea frente a una cara a cara (Caplan, 2003; Chung, 2013) que favorecería y fortalecería vínculos y sentimientos de apego en las comunidades locales (Ei Chew, LaRose, Steinfield, y Velasquez, 2011).

Con respecto al tiempo de conexión, más del 80\% de los menores de varios países (Suecia, Bulgaria, Estonia, Dinamarca, Noruega y Holanda) que utilizan Internet lo hacen a diario, porcentaje que se reduce en otros países (en España hay estudios que lo sitúan en el 68\%). Estas cifras suponen que el uso de Internet diario de un menor europeo entre 9 y 16 años se sitúa en 88 minutos al día (en España en 71 minutos) (Bricolo, Gentile, Smelser, y Serpelloni, 2007; Fundación Pfizer, 2009; Garmendia, Garitaonandia, Martínez, y Casado, 2011). Otros segmentos de edad muestran resultados similares: las personas inscritas en programas universitarios de mayores se conectan a Internet frecuentemente, a diario o entre dos o tres veces por semana (Loscertales Abril, Martínez Pecino, y Cabecinhas, 2011).

No obstante, no se puede establecer una correspondencia entre cantidad de horas de conexión y uso problemático de Internet (Griffiths, 2010); por ejemplo, algunos trabajadores o estudiantes deben estar conectados por cuestiones laborales o de estudios un considerable número de horas (Munoz-Rivas et al., 2010) y también 
encontramos a internautas que emplean Internet como plataforma para el ocio (leer, informarse, ver documentales y películas, oír música, etc.). Por tanto, el uso problemático de Internet no es cuestión de tiempo; se trata más bien de la incapacidad de controlar el tiempo de conexión, relegando e interfiriendo obligaciones y tareas personales, laborales, familiares, sociales o académicas.

En lo que respecta a las redes sociales, se han convertido actualmente en una vía imprescindible de comunicación e información, de creación de proyectos, de integración de comunidades que de otra forma no existirían, una nueva forma de entretenimiento, información y de acción social colectiva (Campos, 2008; Cheung y Lee, 2010; Flores, 2009) para la mayoría de los internautas.

Porcentajes superiores al $80 \%$ de los internautas españoles afirman ser usuarios habituales de las mismas (INTECO, 2012; The Cocktail Analysis, 2012). En el segmento más joven de la población, la participación en dichas redes es seguramente la actividad con un crecimiento más rápido entre los mismos (Garmendia et al., 2011) y en una tendencia consolidada en sus vías de relación. En lo que respecta al alumnado universitario, según Monge y Olabarri (2011) el 90\% tienen cuenta en alguna red social (estudio de 2010), sobre todo para fines de comunicación e interacción entre pares y la construcción de su ser social (Barker, 2012; Bravo y Rasco, 2013; Horgan y Sweeney, 2012).

\section{Las redes sociales en el ámbito educativo}

El rol del docente ha ido cambiando a lo largo de la historia, fruto de la propia evolución de la misma y de las distintas teorías que van apareciendo acerca del binomio enseñanza-aprendizaje. Hoy día, inmersos como estamos en el empleo de las TIC, sigue siendo fundamental la tarea del profesor. Las clases magistrales son ya cosa del pasado. El docente ya no es mero transmisor de conocimientos a sus alumnos. Ahora, este no reside en él. Su labor debe ser, en consecuencia, la de generar conocimientos en los discentes a través de la adquisición de las competencias básicas.

En esta tarea motivadora, facilitadora, mediadora... en la que debe desarrollar su trabajo el profesor, el uso de las redes sociales cobra un importante papel. El trabajo en grupo, el aprendizaje colaborativo, la interacción entre el alumnado... son los pilares en los que se asienta la utilización de las redes en el proceso formativo.

El uso de las redes sociales en los procesos enseñanza-aprendizaje obliga a un cambio profundo en el docente. Cambio que llevaría implícito la aceptación de su nuevo rol, pero que además obligaría a cambiar la formación inicial de los docentes y su formación continua, para adecuarla a la realidad de los nuevos tiempos. El profesorado debe ser consciente de la necesidad de cambios metodológicos y no aferrarse a procedimientos y estrategias del pasado, no adecuados a las necesidades de la sociedad de nuestros días.

Los roles de los docentes están actualmente cambiando del control a la influencia sutil y/o conformación inicial (Siemens, 2010). El papel del estudiante también ha cambiado de escenario, pasando de una pedagogía centrada en el profesor a nuevas 
situaciones en las que el control se sustituye por la dirección del grupo de pares, el intercambio de colaboración, la autonomía y la responsabilidad de los estudiantes.

Los nuevos tiempos requieren un considerable esfuerzo por parte del profesorado para asumir sus necesidades formativas y de alfabetización digital y llevarlas a cabo. Pero, también es grande el cambio que debe realizar el alumnado. El lema del programa de enriquecimiento instrumental de Feuerstein et al. (1980) tiene absoluta vigencia en la metodología de aprendizaje basada en el uso de las redes sociales "un momento, déjame pensar”.

Por otra parte, el alumno debe ser más autónomo, autosuficiente y capaz de construir sus propios conocimientos, a su ritmo, en función de sus necesidades y capacidad. La enseñanza es más personalizada e individualizada. No es igual para todos. Ya Feuerstein et al. (1980) hablaban de aprendizaje mediado. El planteamiento de estos autores se encuadraba en una propuesta innovadora, orientada hacia "el aprender a pensar" y "aprender a aprender".

El alumno "debe pensar" en la forma de acceder a las fuentes de información soportadas por tecnologías y mostrar competencias tecnológicas que le permitan consumir, usar, producir y compartir información para adquirir el conocimiento.

El estudiante en el contexto de las redes sociales ya no es un sujeto pasivo que almacena información. Tiene que seleccionarla, procesarla, compararla, analizar causas, extraer consecuencias...y adecuarla a su estilo de aprendizaje. Cada uno tiene su propia manera de percibir y procesar, todo ello en función del tipo de información y del contexto.

\section{Web 2.0 y redes sociales}

La aparición de la Web 2.0 en el ámbito educativo hace que los procesos de enseñanza-aprendizaje tengan un carácter más social y personalizable en los estudiantes (Shang et al., 2011), las aplicaciones de la denominada Web 2.0 han alterado muchas de las relaciones de poder tradicionales en la enseñanza. Tareas que antes eran responsabilidad de los docentes están ahora bajo el control de los estudiantes: buscar información, crear espacios de interacción, formar redes de aprendizaje, etc. Los estudiantes pueden acceder a contenido de profesores e investigadores líderes de todo el mundo mediante blogs, wikis, vídeo en línea, podcasts y recursos educativos abiertos.

Está claro que a día de hoy, las redes sociales son una realidad en todos los ámbitos en los que nos movemos, tanto a nivel individual como institucional. Las universidades por tanto no deben dar la espalda a esta realidad e intentar aprovechar en todos los aspectos el potencial de poseen.

Richmond, Rochefort y Hitch (2011) reconocen el impacto limitado que tienen las redes sociales en la enseñanza formal actual. En respuesta a esto, destacan la necesidad de que los educadores adquieran formación en la enseñanza y el aprendizaje en red. En 
particular, argumentan que el desarrollo de las destrezas del cuerpo docente en el uso efectivo de las redes sociales es una responsabilidad institucional.

Por tanto, los objetivos a conseguir por cualquier institución, en este aspecto como en cualquier otro, deberían estar definidos previamente y desarrollarse mediante estrategias didácticas en terrenos conocidos. En consecuencia, a las instituciones de educación superior no les va a quedar más remedio que dar respuesta a estas demandas.

El presente trabajo, realizado con estudiantes universitarios de la Facultad de Ciencias de la Educación de la Universidad de Málaga (España), tiene como objetivos conocer si existe dependencia a Internet entre los mismos y los usos que hacen de las redes sociales.

\section{Diseño y metodología}

El estudio que desarrollamos en la Facultad de Ciencias de la Educación de la Universidad de Málaga, tenía como fin analizar el estado del empleo de las redes sociales por parte del alumnado universitario, así como los posibles malos hábitos y usos problemáticos de las mismas.

Los análisis sobre estas aplicaciones relacionadas con las TIC, son desde el punto de vista de las instituciones educativas bien recibidos, pues suelen dar indicios acerca de las posibles debilidades y fortalezas de las mismas y permiten orientar la toma de decisiones en los planes estratégicos o de calidad que elaboren.

En el momento de analizar los datos obtenidos se procedió, en primer lugar, a la transcripción de todos los datos obtenidos y, tras un estudio en profundidad de estos, se clasificaron y registraron. Con el objetivo de realizar un adecuado análisis de los datos recabados, procedimos de la siguiente manera (Schaffert y Hilzensauer, 2008; Bogdan y Biklen, 1992):

- En una primera etapa se identificaron las unidades de información. En esta la codificación de los datos se realizó mediante la identificación de unidades de información (Bardin, 1986).

- En un segundo momento procedimos a su categorización, las unidades de información se clasificaron de manera inductiva.

- Por último procedimos a la fase de interpretación de los datos, en donde se analizaron las diferentes unidades de información categorizadas. Los resultados se clasificaron en tablas y gráficos para facilitar la interpretación y explicación de los resultados, como veremos a continuación.

\section{Instrumento}

Para proceder a la recogida de información, se preparó un cuestionario "ad hoc" que debía ser cumplimentado de forma telemática y anónima, mediante invitación 
personalizada, por el alumnado de la Facultad de Ciencias de la Educación de la Universidad de Málaga. La recogida de datos se realizó entre enero y abril de 2013.

Estaba formado por un total de 23 ítems en el que se recogían datos personales, recursos utilizados y uso de redes sociales. Uno de los ítem incluía las 9 preguntas del cuestionario de adicción a Internet de Orman (2010). La contestación de las mismas fue mediante Sí o No. Siguiendo las indicaciones originales del autor, el nivel de adicción a Internet se establecería sumando el número de Síes en el conjunto de los nueve ítems que componen la prueba. Un número superior a 7 indicaría problemas de dependencia, 3 o menos mostraría ausencia total de problemas, mientras que una puntuación intermedia indicaría que la persona está en zona de riesgo. En esta investigación se utilizó una adaptación de este instrumento que incluía dos modificaciones: por un lado su traducción (Basile, 2006) y, por otro, una escala likert con 5 opciones (nada, apenas, de forma ocasional, a menudo, siempre), en lugar de la respuesta Sí o No, para aumentar la sensibilidad de las respuestas. Aplicada a estas cuestiones el alfa de Cronbach arroja un valor de 0.86 .

\section{Muestra}

La población objeto de nuestro estudio era el alumnado de la Facultad de Ciencias de la Educación. El universo de la población era de 2.420 estudiantes, tal y como recogían los datos ofrecidos por el Servicio Central de Informática de la Universidad de Málaga.

Para calcular el tamaño de la muestra, se partió de un nivel de confianza del 95\% y un intervalo de confianza del 5\%, con lo que la muestra debería ser como mínimo de 339 sujetos. Se recogieron 344 encuestas, con participación desinteresada y voluntaria. Se utilizó un muestreo estratificado proporcional no aleatorio, que Cohen y Manion (1990) denominan muestreo por cuotas, correspondientes a las cuatro especialidades, preservándose la estructura poblacional a través de la fijación de cuotas. El tratamiento estadístico se realizó utilizando el programa SPSS versión 20.

\begin{tabular}{lccc}
\hline \multirow{2}{*}{ Muestra } & \multicolumn{2}{c}{ Sexo } \\
\cline { 3 - 4 } & & Hombre & Mujer \\
\hline Grado Infantil & 92 & 11 & 81 \\
\hline Grado Primaria & 121 & 41 & 80 \\
\hline Grado Pedagogía & 101 & 27 & 74 \\
\hline Grado Ed. Social & 25 & 14 & 11 \\
\hline Total & 339 & 84 & 255 \\
\hline
\end{tabular}

Tabla 1. Muestra por especialidades y género

Una vez analizados los cuestionarios recibidos y filtrados los primeros resultados, se eliminaron los cuestionarios incompletos, quedando una muestra final de 339 alumnos. De los alumnos encuestados el $24,8 \%$ fueron hombres frente al $75,2 \%$ hombre. Y la edad promedio fue de 21,93 años (DT=3,687). 


\section{Resultados}

Antes de comenzar a abordar los resultados de las encuestas estudiadas, consideramos relevante incidir en algunos datos relacionados con su consumo. Su empleo está altamente extendido entre los universitarios, de hecho el 96,64\% de los encuestados afirman ser usuarios de al menos una red social. Por lo que, los datos y porcentajes que vamos a presentar a continuación están calculados a partir de esa base $(\mathrm{n}=318)$ de usuario de redes sociales.

Los estudiantes encuestados emplean un promedio de 2,82 redes sociales. Entre ellas, las utilizadas por el mayor porcentajes es Facebook (85,2\%), seguida por Twitter $(45,1 \%)$ y Tuenti $(27 \%)$. El resto de redes no suponen resultados reseñables, no alcanzado porcentajes superiores al 5\% (MySpace, Hi5, Flickr, Likedin). La utilización de la red social en cuestión se ha convertido en algo totalmente cotidiano para el alumnado, estando integrado plenamente en su quehacer diario. El número de conexiones diarias es destacable, siendo un $61,4 \%$ el alumnado que afirma conectarse varias veces al día, manifestando que el uso de las redes sociales forma parte de sus hábitos cotidianos $(\mathrm{M}=3,67$. $\mathrm{DT}=1,4)$ (escala Likert donde 1 nada de acuerdo y 5 muy de acuerdo).

Una gran parte del alumnado está bastante tiempo conectado a las diferentes redes sociales desde casa principalmente $(\mathrm{M}=3,98$. $\mathrm{DT}=0,89)$ (escala Likert donde 1 es nada hasta 5 mucho) y en la universidad $(M=3,1$. DT=1,076). Sin embargo, la opción en cualquier lugar $(\mathrm{M}=4,21$. $\mathrm{DT}=0.87)$ es la más elegida por el alumnado, debido a las posibilidades de conexión que poseen hoy en día con los diferentes dispositivos móviles que tienen a su alcance (smartphones, tabletas...). A la pregunta sobre el motivo por el que utilizaban las redes sociales, un $87 \%$ ha contestado que para estar en contacto con sus amigos, seguido del $64,3 \%$ que lo hace para estar al día de lo que ocurre en su entorno y un 18,4\% que lo hace para resolver cuestiones académicas.

\begin{tabular}{lcc}
\hline & Media & Desv. Típ. \\
\hline Navegar & 4,01 & 1,002 \\
\hline Comunicarse, amigos, familia... & 3,97 & 1,203 \\
\hline Consulta del correo & 3,94 & 1,114 \\
\hline Realizar gestiones personales & 3,4 & 1,221 \\
\hline Subir Imágenes & 3,25 & 1,125 \\
\hline Jugar & 3,12 & 1,174 \\
\hline Subir vídeos & 2,87 & 1,165 \\
\hline Construir wiki & 2,73 & 1,244 \\
\hline Gestionar Blog & 2,34 & 1,132 \\
\hline Subir podcast & 2,11 & 1,203 \\
\hline Descargar imágenes, vídeos... & 2,03 & 1,264 \\
\hline
\end{tabular}

Tabla 2. Principales usos de Internet

Los estudiantes fueron igualmente encuestados acerca del uso que hacían de Internet (escala Likert de 1 nada a 5 mucho). Navegar buscando información y comunicarme con compañeros, familia, amigos... fueron los usos más comunes entre 
el alumnado. Por el contrario, la descarga de películas o el uso de podcast no se encontraban entre los más utilizados (Tabla 2).

Pero el empleo de Internet y la utilización que se haga puede llevar consigo malos hábitos que desemboquen en cierta dependencia, los estudiantes fueron preguntados acerca de dichos hábitos (escala Likert de 1 nada a 5 siempre), destacando aquellos alumnos que manifiestan dedicar más tiempo del que deberían estar conectados con objetivos distintos a los que en un principio motivaron su conexión. A pesar de ello, afirman que este motivo no hace que empeoren sus relaciones personales.

\begin{tabular}{lcc}
\hline & Media & $\begin{array}{c}\text { Desv. } \\
\text { Típ. }\end{array}$ \\
\hline $\begin{array}{l}\text { Dedicas más tiempo del que crees que deberías a estar conectado a } \\
\text { Internet con objetivos distintos a los de tu trabajo }\end{array}$ & 2,45 & 1,229 \\
\hline Te resulta duro permanecer alejado de Internet varios días seguidos & 2,40 & 1,293 \\
\hline $\begin{array}{l}\text { Existen contenidos o servicios de Internet a los que encuentras difícil } \\
\text { resistirte }\end{array}$ & 2,27 & 1,170 \\
\hline $\begin{array}{l}\text { Obtienes gran parte de tu bienestar del hecho de estar conectado en } \\
\text { Internet }\end{array}$ & 2,02 & 1,085 \\
\hline Se han quejado tus familiares de las horas que dedicas a Internet & 1,92 & 1,140 \\
\hline Piensas que te sentirás mal si redujeras el tiempo que pasas en Internet & 1,74 & 1,007 \\
\hline Has intentado, sin éxito, reducir su uso & 1,54 & 0,860 \\
\hline Se resienten tus relaciones por estar conectado & 1,51 & 0,806 \\
\hline Tienes problemas para controlar el impulso de conectarte a Internet & 1,03 & 1,007 \\
\hline
\end{tabular}

Tabla 3. Principales problemas al utilizar de Internet

Del mismo modo, se analizó de qué manera el uso de internet estaba influyendo en su actividad académica (escala Likert de 1 nada a 5 mucho), en esta ocasión el alumnado manifiesta que ha aumentado de manera significativa su interacción tanto con sus compañeros como con los profesores, de hecho un 50,3\% afirman que ha mejorado bastante o mucho la interacción con el profesorado y un 60,9\% con sus compañeros.

\begin{tabular}{lcc}
\hline & Media & Desv. Típ. \\
\hline Ha aumentado la interacción con tus compañeros & 3,62 & 1,017 \\
\hline Ha aumentado la interacción con los profesores & 3,40 & 1,067 \\
\hline Ha disminuido el uso de documentos convencionales & 3,32 & 1,203 \\
\hline Ha mejorado mi rendimiento académico & 3,31 & 0,978 \\
\hline Ha aumentado la dependencia de uso de Internet & 3,30 & 1,164 \\
\hline Ha disminuido el tiempo que necesito para hacer trabajos & 3,19 & 1,208 \\
\hline
\end{tabular}

Tabla 4. Influencia del empleo de Internet en su actividad académica 
Acerca de los riesgos que puede tener el uso inadecuado de las redes sociales, el alumnado afirma mayoritariamente conocer las precauciones básicas a tener en cuenta (87\%). Los que sostienen no conocerlos suponen un $13 \%$ del total encuestado.

En relación con los hábitos y prácticas más frecuentes en las redes sociales, nos centramos en un primer lugar en algo tan común como es el tema de las imágenes, las respuestas recogidas acerca del uso que hacían de las mismas pusieron de manifiesto que la mayoría sube imágenes a las redes sociales sin pedir permiso a las personas que puedan aparecer (42,6\%), no llegando al $10 \%$ el alumnado que no lo hace nunca con imágenes suyas o de sus contactos más cercanos.

\begin{tabular}{lcc}
\hline & $\mathbf{\%}$ & $\begin{array}{c}\text { \% } \\
\text { Acumulado }\end{array}$ \\
\hline Nunca subes fotografías tuyas o de tus amigos & 9,6 & 9,6 \\
\hline $\begin{array}{l}\text { Subes algunas fotografías, independientemente de quién } \\
\text { salga en ellas }\end{array}$ & 42,6 & 52,2 \\
\hline $\begin{array}{l}\text { Subes únicamente las fotografías en las que apareces. Si } \\
\text { aparecen otros, les pides permiso antes de publicarlas. }\end{array}$ & 29,3 & 81,5 \\
\hline Subes la mayoría de fotografías que tienes & 18,5 & 100,0 \\
\hline
\end{tabular}

Tabla 5. Hábitos más frecuentes de empleo de imágenes en las redes sociales

En la misma línea que lo anteriormente analizado, el alumnado encuestado afirma que nunca le han pedido permiso para publicar una fotografía suya en las redes sociales (43,6\%), mientras que el $12,1 \%$ sostiene que siempre se lo han pedido.

A pesar de los datos expuestos previamente, el alumnado manifiesta mayoritariamente $(87,2 \%)$ no haber sido objeto de ningún tipo de acoso en le red, si bien, afirma que en ocasiones se han publicado en la redes sociales ciertos aspectos sobre su persona con los que no han estado de acuerdo, siendo únicamente un 1,5\% el que señala haber tomado alguna medida de tipo legal para solucionar dicha situación.

En lo que concierne a las posibilidades educativas de las redes sociales, se plantearon a los encuestados una serie de posibilidades para que seleccionasen las que en su opinión resultaban más productivas desde el punto de vista educativo. Se pretendía que valorasen las posibilidades que para el aprendizaje colaborativo permitían herramientas para compartir archivos, comunicarse entre docentes y alumnos...

Entre los resultados obtenidos destacamos que nuestros informantes manifiestan que utilizan bastante las redes sociales para comunicarse con sus compañeros $(89,3 \%)$, pero pocos las emplean para intentar resolver cuestiones académicas $(21,4 \%)$. Asimismo, valoran las posibilidades que para el aprendizaje en red poseen distintas aplicaciones para compartir documentos, comunicación... que ofrecen las redes sociales, pero sin embargo dichos porcentajes son muy bajos, por lo que podemos deducir que en general no encuentran muchas posibilidades educativas al empleo de redes sociales en educación. 


\begin{tabular}{lc}
\hline & Porcentaje \\
\hline Compartir documentos & $17,2 \%$ \\
\hline Mejorar comunicación con los docentes & $11,6 \%$ \\
\hline Mejorar comunicación con compañeros & $14,5 \%$ \\
\hline Compartir conocimientos & $8,3 \%$ \\
\hline
\end{tabular}

Tabla 6. Posibilidades educativas de las redes sociales

\section{Discusión y conclusiones}

Una vez expuestos los resultados de nuestra investigación, podemos concluir que en general nuestro alumnado posee una buena predisposición al uso de las redes sociales. Los porcentajes de conocimiento y empleo de las mismas son lo suficientemente altos, indicando que están plenamente integradas en su día a día. Un 95,6\% de los alumnos manifiestan tener cuenta en alguna red social y se conectan diariamente un $85,2 \%$, en la misma línea de otros estudios similares (Rodrigues, 2008; Urueña et al., 2011). Con respecto al tipo de redes, se hace un uso mayoritario de algunas redes sociales como Facebook, Twitter o Tuenti, el resto tiene un uso menor en relación con las primeras, como también han puesto de manifiesto otros estudios (Cabero y Marín, 2014).

En relación con los problemas de adicción, hemos constatado que un 2,9\%, según el cuestionario de Orman (2010), presenta dichos problemas, porcentaje muy similar a los datos ofrecidos en otros estudios similares (Hawi, 2012; Kuss, Griffiths y Binder, 2013). No se han constatado problemas de dependencia ni de alteración de las conductas habituales en los encuestados, debido al empleo de las redes sociales a pesar de tener un uso masivo y de que en ocasiones no tomen medidas suficientes de precaución en su hábitos cotidianos de manejo de las redes.

Una de las principales causas por la que los estudiantes no relacionan en empleo de redes sociales con el ámbito académico es debido fundamentalmente a que las instituciones educativas no otorgan a estas el papel fundamental que poseen en el día a día de los alumnos. La universidad en la que nos desenvolvemos sigue, en general, anclada en el empleo de aplicaciones y herramientas de la denominada Web 1.0, a pesar de tener implementados servicios de la Web 2.0, el empleo que se realiza de los mismos no es destacable. Con los resultados obtenidos queda patente que el alumnado emplea la redes sociales de forma mayoritaria, coincidiendo con lo que afirman Gutiérrez, Palacios y Torrego (2010), que afirman que en las instituciones académicas las innovaciones educativas se desarrollan de una manera más lenta que las innovaciones tecnológicas lo hacen en el resto de la sociedad.

En la línea de Flores (2009), el gran inconveniente de las redes sociales radica en que las universidades no asumen el cambio que se está produciendo en la red, en donde los usuarios seleccionan, modifican y comentan información de una manera casi al instante. El papel que Internet ha tenido y está teniendo en el desarrollo de las redes sociales en contextos diversos es fundamental, por tanto, desde el ámbito educativo no podemos dar la espalda a este fenómeno. Aquí somos los docentes los que debemos considerar que somos los principales impulsores de los cambios de modelos que pueden producirse con la entrada en escena de estas tecnologías, coincidiendo con 
Duart (2009) en que la verdadera transformación la encontraremos en la dinámica educativa y en los procesos que se desarrollan en las aulas; no pudiendo dar la espalda el empleo activo y adecuado de la red en las planificaciones docentes.

Tenemos por tanto que intentar canalizar las actitudes hacia todas esas innovaciones tecnológicas que asumen de manera natural los estudiantes, en los programas docentes, con el objetivo de promover la creación de comunidades de aprendizaje a lo largo de toda la vida (Frand, 2000). Todo ello posibilitará que el alumnado adquiera las habilidades necesarias de gestión del conocimiento que resultan fundamentales para emplear los medios de los que hoy disponen (Drexler, 2010).

De ese modo, podremos sostener que como el valor añadido de las redes sociales se basa en el atractivo del acercamiento del aprendizaje formal y no formal, en el aumento de la comunicación entre alumnos, en una cambio de rol de docentes y estudiantes que favorezcan nuevos escenarios educativos, en donde con nuestra prácticas y nuestras actividad diaria hemos de ser capaces de poner en marcha metodologías activas y participativas de las que tanto se hablan en el EEES (Espacio Europeo de Educación Superior). En ello el papel que deben jugar las redes sociales es clave pues nos permitirán el asentamiento de nuevas formas de trabajo colaborativo más atractivas.

Sin embargo, coincidimos con Espuny et al. (2010) en que en la actualidad los modelos didácticos innovadores son poco frecuentes y se suelen fundamentar en sistemas en los que, con pequeñas variaciones, el docente explica y el alumno escucha. García Sans (2008) se lamenta de que los recelos hacia las redes sociales se traduzcan en una escasa investigación acerca de su rendimiento educativo, y de que gran parte del profesorado tiene ese mismo sentimiento de rechazo hacia la tecnología utilizada por los estudiantes, puesto que interfiere en el flujo natural de la enseñanza tradicional (Handley et al., 2007). A pesar de todo, las redes sociales las tenemos presente en nuestro día a día y están cambiando nuestra forma de relacionarnos. La clave está en los docentes, y en la manera en que lo asuman nuestros estudiantes, para que con esta manera de aprender, de investigar y de trabajar permanezcan o no al margen de esta revolución.

Por último, podemos concluir que aún no están plenamente integradas en las instituciones el empleo de las redes sociales, ni que las estudiantes las utilicen como herramienta fundamental para las resolución de cuestiones académicas. Es mayoritaria la utilización de estas como herramientas de comunicación y ocio, mostrando los encuestados muy buena predisposición en su manejo, por lo que su correcta integración en los entornos educativos podría favorecer la consecución de los objetivos planteados por el EEES.

\section{Referencias bibliográficas}

BARDIN, L. (1986). Análisis de contenido. Madrid: Akal

BARKER, V. (2012). A Generational Comparison of Social Networking Site Use: The Influence of Age and Social Identity. The International Journal of Aging and Human Development, 74(2), 163-187. doi:10.2190/AG.74.2.d 
BASILE, H. (2006). Adicción a Internet: Ciberadicción. Revista Argentina de Clínica Neuropsiquiátrica, 13(2), (pp. 74-90).

BOGDAN, R. y BIKLEN, S. K. (1992). Investigación cualitativa de la educación. Needham Heights, MA: Allyn and Bacon

BRAVO, C. B., y RASCO, F. A. (2013). Interacciones de los jóvenes andaluces en las redes sociales - Interactions of Young Andalusian People inside Social Networks. Revista Comunicar, XX(40), 25-30. doi:10.3916/C40-2013-02-02

BRICOLO, F., GENTILE, D. A., SMELSER, R. L., y SERPELLONI, G. (2007). Use of the Computer and Internet among Italian Families: First National Study. CyberPsychology \& Behavior, 10(6), 789-798. doi:10.1089/cpb.2007.9952

CABERO, J. y MARÍN, V. (2014). Posibilidades educativas de las redes sociales y el trabajo en grupo. Percepciones de los alumnos universitarios. Comunicar, 42, (pp.165-172). (DOI: 10.3916/C42-2014-16).

CAMPOS, F. (2008). Las redes sociales trastocan los modelos de los medios de comunicación tradicionales. Revista Latina de Comunicación Social, 63, 287-293. doi:10.4185/RLCS-63-2008-767-287-293

CAPLAN, S. E. (2003). Preference for Online Social Interaction A Theory of Problematic Internet Use and Psychosocial Well-Being. Communication Research, 30(6), 625-648.

CARBONELL, X., FÚSTER, H., CHAMARRO, A., y OBERST, U. (2012). Adicción a Internet y móvil: una revisión de estudios empíricos españoles. Papeles del Psicólogo, 33(2), 82-89.

CHEUNG, C. M., y Lee, M. K. (2010). A theoretical model of intentional social action in online social networks. Decision Support Systems, 49(1), 24-30.

CHUNG, J. E. (2013). Social interaction in online support groups: Preference for online social interaction over offline social interaction. Computers in Human Behavior, 29(4), 1408-1414. doi:10.1016/j.chb.2013.01.019

COHEN, L. y MANION, L. (1990). Métodos de investigación educativa. Madrid: La Muralla

DREXLER, W. (2010). The networked student model for construction of personal learning environments: Balancing teacher control and student autonomy. Australasian Journal of Educational Technology, 26(3), 369-385. Disponible en http://www.ascilite.org.au/ajet/ajet26/drexler.html, 4 de marzo de 2014.

DUART, J. M. (2009). Internet, redes sociales y educación. RUSC. Revista de Universidad y Sociedad del Conocimiento, 6(1), (pp. 1-2) 
EI CHEW, H., LAROSE, R., STEINFIELD, C., Y VELASQUEZ, A. (2011). The Use of Online Social Networking by Rural Youth and Its Effects on Community Attachment. Information, Communication \& Society, 14(5), 726-747. doi:10.1080/1369118X.2010.539243

ESPUNY, C. et al. (2011). Actitudes y expectativas del uso educativo de las redes sociales en los alumnos universitarios. Revista de Universidad y Sociedad del Conocimiento (RUSC), 8(1), (pp. 171-185). UOC. Recuperado de http://rusc.uoc.edu/ojs/index.php/rusc/article/view/v8n1-espuny-gonzalez-lleixagisbert/v8n1-espuny-gonzalez-lleixa-gisbert, 29 de junio de 2014.

FEUERSTEIN, R. et al. (1980). Instrumental Enrichment: an intervention program for cognitive modifiability. Baltimore: University Park Press.

FLORES, J. M. (2009). Nuevos modelos de comunicación, perfiles y tendencias en redes sociales. Comunicar. Revista Científica de Educomunicación, 17(33), (pp. 7381)

FRAND, J. L. (2000). The Information-Age Mindset: Changes in Students and Implications for Higher Education. EDUCAUSE Review, 35(5), 15-24. Recuperado de www.educause.edu/apps/er/erm00/articles005/erm0051.pdf, 22 de junio de 2014.

FUNDACIÓN PfIzer. (2009). La Juventud y las Redes Sociales en Internet. Recuperado a partir de https://www.fundacionpfizer.org/sites/default/files/pdf/INFORME_FINAL_Encuest a_Juventud_y_Redes_Sociales.pdf. 1 de marzo de 2014.

GARCÍA SANS, A. (2008). Las redes sociales como herramientas para el aprendizaje colaborativo: una experiencia con Facebook. En: Actas del XIII Congreso Internacional en Tecnologías para la Educación y el Conocimiento: la Web 2.0. Madrid: UNED.

GARMENDIA, M., GARITAONANDIA, C., MARTÍNEZ, G., Y CASADO, M. Á. (2011). Riesgos y seguridad en internet: Los menores españoles en el contexto europeo. Bilbao: Servicio Editorial de la Universidad del País Vasco. Recuperado a partir http://www2.lse.ac.uk/media@lse/research/EUKidsOnline/EU\%20Kids\%20II\%20 \%282009-11\%29/National\%20reports/Spanish\%20report.pdf. 29 de junio de 2014.

GRACIA, M. DE, VIGO, M., FERNÁNDEZ, M. J., Y MARCÓ, M. (2002). Problemas conductuales relacionados con el uso de Internet: Un estudio exploratorio. Anales de Psicología, 18(2), 273-292.

GRIFFITHS, M. D. (2010). The Role of Context in Online Gaming Excess and Addiction: Some Case Study Evidence. International Journal of Mental Health and Addiction, 8(1), 119-125. doi:10.1007/s11469-009-9229-х

GUTIÉRREZ, A.; PALACIOS, A. Y TORREGO, L. (2010). Tribus digitales en las aulas universitarias. Comunicar, 34, (pp. 173-181). 
HANDELEY, C. et al. (2007). Out of the Classroom \& into the Boardroom. Higher Education Consortium. Microsoft

HAWI, N. S. (2012). Internet addiction among adolescents in Lebanon. Computers in Human Behavior, 28(3), 1044-1053. doi:10.1016/j.chb.2012.01.007

HORGAN, A., y SWEENEY, J. (2012). University Students' Online Habits and Their Use of the Internet for Health Information. CIN: Computers, Informatics, Nursing, 30(8), 402-408. doi:10.1097/NXN.0b013e3182510703

INTECO. (2012). Estudio sobre la seguridad de la información y la e-confianza de los hogares españoles, 1er cuatrimestre de 2012 (18 ${ }^{a}$ oleada) (p. 96). Instituto Nacional de Tecnologías de la Comunicación. Recuperado a partir de http://www.inteco.es/Estudios/Estudio_hogares_1C2012. 11 de mayo de 2014.

KUSS, D., GRIFFITHS, M. Y BINDER, J. (2013). 525 - Online on campus: internet addiction and personality in english university students. European Psychiatry, 28, Supplement 1, 1. doi:10.1016/S0924-9338(13)75828-0

LOSCERTALES ABRIL, F., MARTÍNEZ PECINO, R., Y CABECINHAS, R. (2011). University Senior Students on the Web. Comunicar, 19(37), 89-95. doi:10.3916/C37-2011-02-09

MONGE, S., Y OLABARRI, E. (2011). Los alumnos de la UPV/EHU frente a Tuenti y Facebook: usos y percepciones. Revista Latina de comunicación social, (66), 4.

MUNOZ-RIVAS, M. J., FERNANDEZ, L., Y GAMEZ-GUADIX, M. (2010). Analysis of the Indicators of Pathological Internet Use in Spanish University Students. Spanish Journal of Psychology, 13(2), 697-707.

PANCKHURST, R. Y MARSH, D. (2011). Utilización de redes sociales para la práctica pedagógica en la enseñanza superior impartida en Francia: perspectivas del educador y del estudiante. Revista de Universidad y Sociedad del Conocimiento (RUSC), 8(1), (pp. 233-252). UOC. Recuperado de http://rusc.uoc.edu/ojs/index.php/rusc/article/view/v8n1-panckhurst-marsh/v8n1panckhurst-marsh. 19 de marzo de 2014.

RICHMOND, N., ROCHEFORT, B. Y HITCH, L.P. (2011). Using Social Networking Sites During the Career Management Process, en Wankel, L.A y Wankel, C. (Eds.). Higher Education Administration with Social Media: Including Applications in Student Affairs, Enrolment Management, Alumni Affairs, and Career Centers. Bingley (UK): Emerald.

RODRÍGUEZ, E., MEJÍAS, I., Y MENÉNDEZ, T. (2012). Consumo televisivo, series e Internet Un estudio sobre la población adoloscente de Madrid. Madrid: FAD. Recuperado a partir de http://www.fad.es/node/584. 5 de julio de 2014.

SHANG, S.S. et al. (2011). Understanding Web 2.0 Service Models: a KnowledgeCreating Perspective. Information \& Management, 48, (pp. 178-184). DOI: http://dx.doi.org/10.1016/j.im.-20 11.01.005. 
THE COCKTAIL ANALYSIS. (2012). $4^{a}$ Oleada Observatorio de Redes Sociales: Las marcas empiezan a encontrar límites - The Cocktail Analysis. Recuperado a partir de http://tcanalysis.com/blog/posts/las-marcas-empiezan-a-encontrar-limitesen-la-utilizacion-de-las-redes-sociales. 12 de junio de 2014.

WIDYANTO, L., Y GRIFFITHS, M. (2006). 'Internet addiction': a critical review. International Journal of Mental Health and Addiction, 4(1), 31-51.

YOUNG, K. S. (1998). Internet Addiction: The Emergence of a New Clinical Disorder. CyberPsychology \& Behavior, 1(3), 237-244. doi:10.1089/cpb.1998.1.237

\section{Referencias digitales}

ORMAN, M. C. (2010). Internet Stress Survey. [formato html] http://www.stresscure.com/hrn/addiction.html (consultado 19 de junio de 2014).

RODRIGUES, R. (2008). Acceso y uso de Internet por parte del estudiantado de la UCA. [formato pdf] http://juanortega.info/wp-content/uploads/2008/11/informeinvestigacion-uso-internet1.pdf (consultado 12 de julio de 2014).

SCHAFFERT, S. Y HILZENSAUER, W. (2008). On the way towards Personal Learning Environments: Seven crucial aspects. Elearningpapers, 9. [formato pdf] http://www.elearningeuropa.info/files/media/media15971.pdf, (consultado 16 de mayo de 2014).

SIEMENS, G. (2010). Teaching in Social and Technological Networks. Connectivism. [formato html] http://www.connectivism.ca/?p=220 (consultado 21 de mayo de 2014).

URUEÑA, A. et al. (2011). Las Redes Sociales en Internet. MIET. [formato pdf] http://www.ontsi.red.es/ontsi/sites/default/files/redes_sociales-documento_0.pdf (consultado 9 de junio de 2014). 


\section{Correspondencia con los autores}

José SÁNCHEZ-RODRÍGUEZ

Facultad de Ciencias de la Educación

Campus de Teatinos s/n

29071 Málaga

Teléfono: 952132558

e-mail: josesanchez@uma.es

Julio RUIZ-PALMERO

Facultad de Ciencias de la Educación

Campus de Teatinos s/n

29071 Málaga

Teléfono: 952132944

e-mail: julio@uma.es

Enrique SÁNCHEZ-RIVAS

Facultad de Ciencias de la Educación

Campus de Teatinos s/n

29071 Málaga

Teléfono: 952137402

e-mail: enriquesr@uma.es 